\title{
Éditorial
}

\section{A la recherche de l'indétectable, la toxicologie médico-légale progresse au rythme des sciences analytiques}

\section{Testing for the undetectable: forensic toxicology is making progress at the rate of analytical sciences}

Pascal KINTZ

Institut de Médecine Légale, 11, rue Humann, 67000 Strasbourg - pascal.kintz@ wanadoo.fr

Présentée comme l'examen complémentaire ayant le meilleur rendement diagnostic après une autopsie, la toxicologie médico-légale (en particulier dans le cadre de la recherche des causes de la mort) a bénéficié ces dernières années des progrès immenses de la science analytique.

De même, l'accroissement de demandes d'expertises toxicologiques chez le vivant (conduite automobile sous influence, dopage, soumission chimique ...) rend nécessaire une démarche constante vers l'amélioration du rendu des résultats et donc de leur interprétation en tenant compte de l'ensemble des données médicolégales.

Il convient, surtout dans le cadre d'affaires criminelles, de faire les prélèvements en double (éventuelle contreexpertise). Ces échantillons devraient être scellés, puis immédiatement conservés au froid $\left(+4^{\circ} \mathrm{C}\right.$ ou $\left.-20^{\circ} \mathrm{C}\right)$, à l'exclusion des cheveux qui seront conservés au sec, à température ambiante. Chaque prélèvement doit être soigneusement identifié.

La pratique de l'expertise médico-légale nous a permis de distinguer deux types de prélèvements à visée toxicologique : des prélèvements obligatoires et des prélèvements facultatifs (à recueillir lorsque certains prélèvements obligatoires sont manquants ou qu'il peut y avoir un intérêt scientifique). Les prélèvements obligatoires sont au nombre de sept : sang cardiaque, sang périphérique, urines, humeur vitrée, cheveux, contenu gastrique, poumon. En cas de levée de corps, seuls les 5 premiers prélèvements sont disponibles. Les prélèvements facultatifs sont la bile, les viscères, les écouvillons naso-pharyngés et les liquides de putréfaction.

La fenêtre de détection des xénobiotiques a pu être complètement modifiée par l'introduction du cheveu dans l'arsenal analytique. Ce tissu possède la propriété unique d'être le marqueur des expositions répétées ou chroniques, permettant en outre d'établir le profil de consommation à long terme et son évolution. Dans la pratique, l'analyse sanguine ou urinaire et l'analyse des cheveux s'avèrent plutôt complémentaires, le sang ou les urines permettant de caractériser un usage ponctuel et les cheveux une exposition cumulée. 
Les cheveux sont généralement prélevés en vertex postérieur. Une mèche de 80 cheveux (diamètre d'un crayon à papier) est suffisante. Les mèches doivent être prélevées le plus près de la peau, coupée au ciseau (ne pas arracher) et orientée racine extrémité au moyen d'une cordelette, fixée $1 \mathrm{~cm}$ au dessus du niveau de la racine. La conservation est aisée ; elle s'effectue en tube sec ou dans une enveloppe, à température ambiante.

La décennie écoulée a vu la mise sur le marché de systèmes analytiques de plus en plus performants tant sur le plan de la sensibilité que sur celui de la spécificité. La spectrométrie de masse est désormais la règle absolue en matière d'expertise toxicologique.

Compte tenu des spécificités de chaque situation médico-légale, l'expert judiciaire se doit de disposer d'un parc analytique important. Le Tableau 1 détaille le matériel nécessaire à la bonne exécution des investigations toxicologiques en fonction des diverses demandes.

A cette liste de matériel, il convient de rajouter un système de chromatographie en phase gazeuse couplée à la spectrométrie de masse avec introduction par espace de tête (HS/GC/MS) pour la recherche des substances volatiles (solvant, gaz, poppers...) ou la confirmation absolue des cyanures ou du monoxyde de carbone et un système d'ICP/MS pour la détermination des métaux et métalloïdes.

L'expertise toxicologique se doit d'établir, confirmer ou infirmer le diagnostic d'intoxication, basée sur : des échantillons biologiques de qualité, un personnel suffisant et compétant, des équipements analytiques performants et coûteux, une interprétation rigoureuse des résultats.

Dès le début de la chaîne de l'analyse, il apparaît évident que la qualité du prélèvement va conditionner celle des résultats. Dans le cadre de l'optimisation des échantillons biologiques, il y a lieu de considérer la nature des échantillons à prélever, leur délai d'acheminement au laboratoire, leur modalité de conservation sur site et les informations indispensables avec la demande.

En parallèle, l'analyste se voit confronter à des difficultés spécifiques comme de disposer de prélèvements corrects et d'un matériel adapté à sa mission, de s'informer sur les nouvelles substances, de trouver de la bibliographie et surtout de disposer des substances et de leurs métabolites comme référence.

Tableau I : Quel matériel pour quelle analyse?

\begin{tabular}{|c|c|c|c|c|c|}
\hline Situation médico-légale & EIA & LC-DAD & GC-MS & LC-MS & GLC-MS/MS \\
\hline Suivi d'un traitement de substitution & $\mathrm{x}$ & & $\mathrm{x}$ & $\mathrm{x}$ & \\
\hline Recherche des causes de la mort & $\mathrm{x}$ & $\mathrm{x}$ & $\mathrm{x}$ & $\mathrm{x}$ & \\
\hline Conduite automobile & & $\mathrm{x}$ & $\mathrm{x}$ & $\mathrm{x}$ & \\
\hline Crime ou délit & $\mathrm{x}$ & & $\mathrm{x}$ & $\mathrm{x}$ & $\mathrm{x}$ \\
\hline Dopage & & & $\mathrm{x}$ & $\mathrm{x}$ & \\
\hline Soumission chimique & & & & $\mathrm{x}$ \\
\hline
\end{tabular}

Abréviations

EIA : automate d'immuno-chimie

LC-DAD : chromatographie en phase liquide couplée à un détecteur à barette de diodes

GC-MS : chromatographie en phase gazeuse couplée à un spectromètre de masse

LC-MS : chromatographie en phase liquide couplée à un spectromètre de masse

GLC-MS/MS : chromatographie en phase gazeuse ou liquide couplée à un spectromètre de masse en tandem. 